\title{
Diseño y validación de un instrumento observacional para la valoración de acciones tácticas ofensivas en fútbol - vatof Design and validation of an observational instrument for the evaluation of offensive tactical actions in football - vatof \\ *Sergio Andrés Caicedo Parada, **Mayra Andrea Calderón Vargas \\ *Universidad de Murcia (España), **Institución Superior de Educación Rural ISER (Colombia)
}

\begin{abstract}
Resumen. Los indicadores de desempeño cada vez ganan más importancia dentro de la toma de decisiones informada en deportes de competición, que permitan sintetizar e interpretar las interacciones de juego en el fútbol. El objetivo de este estudio es diseñar, validar y probar confiabilidad de un instrumento de observación dentro de la dinámica del juego, permitiendo comprender el comportamiento, categorización y registro de las acciones tácticas en fútbol. Asimismo, y de acuerdo con la finalidad del instrumento, se enfoca la mirada en la dimensión de rendimiento, pues concretamente, ésta es la que resume el éxito de la acción táctica ofensiva, manteniendo su interrelación con las otras dimensiones, pues en conjunto configuran el cómo, cuándo y dónde se ejecutan las jugadas. La validez del contenido fue establecida por 10 jueces con criterios de escogencia: (a) ser doctor en Ciencias de la Actividad Física y Deporte, (b) 5 años de experiencia, (c) tener licencia de entrenador y haberlo sido durante mínimo 3 años, dando como unidades de análisis finalizadas 7 dimensiones y 40 criterios. Se utilizó la metodología DELPHI de juicio de expertos. El índice V de Aiken fue de 0.93 para determinar la pertinencia del contenido, la índice kappa fue de 1.00 análisis intraobservador para probar la confiabilidad de la herramienta de observación. Estos procedimientos indican que la herramienta contiene información válida y confiable para ser replicada en otros estudios y analizar las acciones de juego a la ofensiva en el fútbol.
\end{abstract}

Palabras clave: deporte colectivo, evaluación, metodología observacional, rendimiento, fútbol.

Summary. Performance indicators are becoming more and more important in informed decision making in competitive sports, as they allow to synthesize and interpret game interactions in football. The objective of this study is to design, validate, and test the reliability of an observation instrument within the dynamics of the game, allowing to understand behaviors, categorization, and recording of tactical actions in football. Likewise, and according to the purpose of the instrument, attention is turned towards the performance dimension, since that is the factor concretely establishing the success of any offensive tactical action, maintaining its interrelation with the other dimensions, since they as a whole configure how, when, and where plays are executed. Content validity was established by 10 judges selected on the basis of the following criteria: (a) to be doctor in Sciences of the Physical Activity and Sport, (b) 5 years of experience, (c) to hold a trainer license for at least 3 years; resulting in 7 dimensions and 40 criteria by the end of the analysis. The experts' evaluation DELPHI methodology was used. Aiken V index, determining the relevance of the content, was 0.93 ; intra-observer analysis' kappa index, which tested the reliability of the observation tool, was at 1.00 . These procedures indicate that the tool contains valid and reliable information to be replicated in other studies and to analyze the actions of offensive plays in football.

Keywords: collective sport, evaluation, observational methodology, performance, football.

\section{Introducción}

En un mundo tan competitivo como el actual, el fútbol moderno enfrenta múltiples desafíos de acuerdo con la naturaleza y dinámica del juego, motivando a los investigadores en identificar patrones e indicadores de rendimiento (Dufour, Phillips \& Ernwein, 2017; Hughes, 2003), que puedan brindar a los entrenadores información útil sobre el análisis individual y colectivo de sus jugadores (Gudmundsson \& Horton, 2017). La cuantificación de las acciones tácticas ofensivas, la frecuencia con que se ejecutan de manera individual y grupal, así como el inicio y finalización, la identificación dentro de zonas del terreno de juego, tanto propias como contrarias, son unidades básicas dentro del análisis de la calidad del dato. Esto a partir de un análisis de observación depurado, donde desarrolle una perspectiva dinámica ecológica (Lozano, Camerino \& Hileno, 2016) que permita la relación existente entre acciones y situaciones tácticas enmarcadas en la percepción, identificación, decisión y ejecución.

En este sentido (Araújo, Teques, Hernández-Mendo, Reigal \& Anguera, 2016; Davids, Button \& Bennett, 2008;

Fecha recepción: 23-11-19. Fecha de aceptación: 02-02-20 Sergio Andrés Caicedo Parada sandres.caicedo@um.es
Lozano, et al., 2016), afirman que la toma de decisión es un proceso de gran complejidad que tiene que ver al menos con tres factores: 1) las características individuales de los deportistas; 2) las condiciones de la tarea que se debe resolver y 3) las características del entorno de actuación. Análisis de desempeño que, a partir de la información recolectada con la herramienta, permite al equipo técnico implementar estrategias pedagógicas y tácticas con la simulación de situaciones competitivas, buscando que los deportistas puedan potenciar sus fortalezas, minimizar sus debilidades y contrarrestar efectivamente las acciones del rival.

En el análisis de la metodología observacional existen diferentes instrumentos que tienen como fin recolectar y proporcionar información de diferentes variables en el aspecto deportivo (Anguera, 2003; Anguera \& Hernández, 2015; Fabra, Balaguer, Tomás, Smith y Duda, 2018; Maneiro, Losada, Casal, \& Ardá, 2018; Nadal, Serna, Nuviala \& Falcón, 2018), asimismo y de acuerdo con (Anguera \& Mendo, 2013) la importancia de la observación en el ámbito deportivo desde una vertiente procedimental, radica en que es la «única metodología científica que permite la recogida de datos directamente de los participantes en entrenamientos y competición a partir de la captación de la información perceptible (p. 136)». 
En esta línea de razonamiento encontramos instrumentos de observación ad hoc, se utilizan para registrar eventos sobre los comportamientos realizados por los jugadores para ser interpretada de acuerdo a la realidad de juego en su contexto natural, intentando responder a las necesidades de un problema específico para mejorar su rendimiento (Anguera, Camerino, Castañer, Sánchez-Algarra \& Onwuegbuzie, 2017; Cuerva \& Ruano 2017; Chacón-Moscoso, et al., 2018; Dios, Jiménez, García \& García, 2018; Morillo, Reigal, HernándezMendo, Montaña \& Morales-Sánchez, 2017; Serna, Muñoz, Hileno, Solsona \& Sáez de Ocáriz Granja, 2017). Por otra parte, encontramos en la literatura softwares especializados de observación similares como: (a) el SOFBAS (actualmente en su segunda versión) desarrollado inicialmente por (Castellano, 2000); (b) SOF (actualmente en su quinta versión), elaborado en su primera versión por (Anguera, et al., 2003); (c) FUT-SAT, realizado por (Costa, Garganta, Greco, Mesquita \& Maia, 2011); (d) SoccerEye, desarrollado por (Barreira, Garganta, Prudente \& Anguera, 2012) y REOFUT, elaborado por (González, 2013), herramientas específicas al rendimiento en fútbol. Estas herramientas reúnen información sobre el inicio de la fase ofensiva, la posesión del balón y finalización de la acción, analizan acciones aisladas, secuencias lineales (Sarmento et al., 2016) que empiezan en la recuperación del móvil y culminan en la concreción del gol, no integran la acción en el contexto de juego con su dinámica habitual.

Sin embargo, se hace necesario un instrumento de observación que permita detallar variables confiables e informativas de rendimiento (Mackenzie \& Cushion, 2013), analizar acciones discretas en escenarios concretos (Travassos, Davids, Araujo \& Esteves, 2013), que analice la continuidad de las acciones a la ofensiva de cada jugador con balón, sin separarlas del juego, sin dificultad en identificar las acciones en conjunto, desde el portero como posible iniciador de la acción, hasta el delantero como posible finalizador de la fase ofensiva. Sobre las bases de las ideas expuestas, la dinámica del juego necesita un instrumento que permita sintetizar e interpretar las interacciones en curso, permitiendo comprender el cómo y porqué en el rendimiento colectivo, detectando y explotando comportamientos funcionales y patrones de equipo acorde a la complejidad, identificando posibilidades de acción más funcionales (Caicedo, Vera \& Ortega, 2018). Constituyendo así, un perfil ideal para predecir el comportamiento a futuro en una determinada situación donde se desempeñe con éxito la sincronía de sus jugadores a partir de la situación numérica.

Por lo tanto, el objetivo de este estudio es diseñar, validar y probar confiabilidad de un instrumento de observación que permita comprender el comportamiento categorización y registro de las acciones tácticas en fútbol.

\section{Materiales y Métodos}

\section{Diseño}

Este estudio utilizó la metodología observacional, definida a su vez como nomotética, de monitoreo y multidimensional (Anguera \& Mendo, 2013). El formato de campo mixto del instrumento diseñado empleó un sistema de categorías (Anguera y Hernández Mendo, 2013); la versión final se conformó con siete dimensiones y 40 núcleos cate- góricos que contemplan el seguimiento espacio temporal. Para el análisis general el diseño tuvo en cuenta tres facetas: observador, dimensiones y núcleos categóricos permitiendo el análisis directo, secuencial, sistemático y objetivo de las conductas de los sujetos estudiados.

\section{Participantes}

La muestra estudiada la integran 14 partidos de los playoffs del Fútbol Profesional Colombiano, de los cuales cuatro se jugaron ida y vuelta (8 partidos), dos partidos de ida y vuelta durante la fase semifinal (4 partidos) y un partido ida y vuelta en la final del torneo ( 2 partidos), para un total de 14 partidos jugados.

\section{Instrumento}

VATOF (Herramienta de Observación para la Valoración de Acciones Tácticas Ofensivas en Fútbol), es un sistema de observación adaptado que permite analizar detalladamente cómo fueron ejecutadas las acciones tácticas ofensivas; además de ser una herramienta práctica para la recolección de información clara y sistemática, permite definir parámetros de acuerdo a la obtención y control de los datos para su respectivo análisis (Anguera, Blanco-Villaseñor, Losada y Portell, 2018), a la vez que cuantifica las acciones ejecutadas en periodos de tiempo determinados, permitiendo la evolución propia y adaptación al rival.

Con un total de siete (7) dimensiones y cuarenta (40) criterios - núcleos categóricos, el VATOF clasifica las acciones tácticas ofensivas temporales, espaciales y modales (Ballesteros, Peñas, Eiras, Martínez \& Lago, 2012) en los siguientes criterios: intervalos de tiempo (minuto de juego), inicio de juego (recuperación, reanudación), tipo de acción (pase, control, conducción, regate, tiro a portería), tipo de $\mathrm{ABP}$ (tiro libre directo e indirecto, tiro de esquina, tiro penal, saque de banda, saque de meta, saque inicial), rendimiento (disparo, disparo a portería o gol), tipo de jugada (individual o grupal), zona de influencia (carril izquierdo, central o derecho), nivel de interacción (superioridad, igualdad o inferioridad numérica), zonas del terreno de juego (inicio y finalización de las zonas: defensiva retrasada, defensiva adelantada, gestación retrasada, gestación adelantada, finalización retrasada, finalización adelantada) y el tiempo (duración de la jugada), como afirman diferentes autores (Mackenzie y Cushion, 2013; Sarmento et al., 2018); buscando establecer esquemas de juego con características y posibilidades esenciales como: la observación del rendimiento (análisis actual) y la observación del desarrollo (análisis evolutivo), lo que facilita registrar e identificar la diversificación de los comportamientos observables tanto propios como contrarios, permitiendo detallar individual y colectivamente el análisis de redes en función a la posición del jugador dentro del terreno de juego. Las categorías establecidas fueron exhaustivas y mutuamente excluyentes (Anguera y Mendo, 2013). Para la versión final del instrumento se diseñó un sistema lógico y completo de códigos a partir de las primeras letras del concepto para su fácil identificación (Sabio, Guerra, Cabedo, Solá \& Argudo, 2018). En las tablas (1, 2, 3, 4, 5, 6, 7) se registra la lista de grupos categóricos, códigos y definición de cada una. 
Tabla 1

Criterios, Codificación de las del instrumento de Valoración de acciones tácticas ofensivas VATOF

\begin{tabular}{|c|c|c|}
\hline \multicolumn{2}{|c|}{ nensión. Tipo de Acción } & \multirow[b]{2}{*}{ Definición } \\
\hline Criterio & Código & \\
\hline Recuperación & $\mathrm{RC}$ & $\begin{array}{l}\text { Acción de juego que limita los movimientos del rival en posesión } \\
\text { del balón, con el fin de obtener el control del mismo e iniciar un } \\
\text { ataque o ejecutar un tiro a portería. }\end{array}$ \\
\hline eanudación & RA & $\begin{array}{l}\text { Reactivación del partido mediante acciones a balón parado, que se } \\
\text { ejecutan posterior a una interrupción del juego y dan paso a la } \\
\text { creación de un nuevo ataque ofensivo. }\end{array}$ \\
\hline Pase & $P$ & $\begin{array}{l}\text { Acción de transferencia del móvil entre jugadores de un mismo } \\
\text { equipo, para la creación colectiva de situaciones de juego o a la } \\
\text { conservación del balón. }\end{array}$ \\
\hline ntrol & CT & $\begin{array}{l}\text { Capacidad del jugador para dominar el balón, permitiendo } \\
\text { asegurar la posición y las condiciones para la acción posterior. } \\
\text { Es la acción técnica que le permite al jugador manejar el balón de }\end{array}$ \\
\hline onducción & $\mathrm{CD}$ & $\begin{array}{l}\text { una posición a otra sin que se ponga en riesgo la posesión del } \\
\text { mismo. }\end{array}$ \\
\hline gate & $\mathrm{R}$ & $\begin{array}{l}\text { Acción técnica que le permite al jugador desbordar uno o más } \\
\text { adversarios conservando el control del balón. }\end{array}$ \\
\hline Tiro a portería & ТР & $\begin{array}{l}\text { Es la acción técnica que le permite al jugador finalizar la acción } \\
\text { ofensiva con remate a portería, con o sin presencia del adversario. }\end{array}$ \\
\hline
\end{tabular}

\begin{tabular}{l} 
Tiro a portería $\quad$ TP $\quad \begin{array}{l}\text { Es la acción técnica que le permite al jugador finalizar la acción } \\
\text { ofensiva con remate a porteria, con o sin presencia del adversario. }\end{array}$ \\
\hline
\end{tabular}

Tabla 2

\begin{tabular}{lcll} 
Dimensión. Tipo de A.B.P. Acción a balón parado \\
\hline Tiro libre & Directo & TLD & Es el conjunto de acciones previamente \\
& Indirecto & TLI & planeadas con el objetivo de sorprender \\
Tiro de Esquina & TE & TP & (proceso ofensivo) o contrarrestar (proceso \\
Tiro Penal & SB defensivo) al adversario, dan el inicio o la \\
Saque de Banda & SM continuidad del juego por medio de una acción \\
Saque de Meta & SI a balón parado. \\
Saque Inicial &
\end{tabular}

Tabla 3

Dimensión de Rendimiento

Disparo D Se caracteriza por ser un remate sin ninguna dirección clara hacia la

Disparo a DP Se caracteriza por terminar en manos del portero, en un rechazo de un

Portería DP jugador propio o contrario o con un golpe en poste.

Gol G Concreción del objetivo de este deporte, vulnerar la portería adversaria.

Tabla 4

Dimensión. Tipo de Jugada

\begin{tabular}{lll}
$\begin{array}{ll}\text { Individual } \\
\text { Grupal }\end{array}$ & 1 & Ejecución individual de un ataque. \\
\hline
\end{tabular}

Tabla 5

Dimensión. Zona de Influencia

\begin{tabular}{|c|c|c|c|}
\hline \multirow{2}{*}{ Derecha } & Inicia Derecha & ID & \multirow{6}{*}{$\begin{array}{l}\text { Esta dimensión permite identificar el carril } \\
\text { (derecho, centro, izquierdo) en el que se } \\
\text { inicia y finaliza una acción de juego. }\end{array}$} \\
\hline & Finaliza Derecha & FD & \\
\hline \multirow{2}{*}{ Centro } & Inicia Centro & IC & \\
\hline & Finaliza Centro & $\mathrm{FC}$ & \\
\hline \multirow[b]{2}{*}{ Izquierda } & Inicia Izquierda & II & \\
\hline & $\begin{array}{l}\text { Finaliza } \\
\text { Izquierda }\end{array}$ & FI & \\
\hline
\end{tabular}

Tabla 6

Dimensión. Nivel de Interacción

Superioridad Está determinada por la participación de un mayor número de Superioridad + jugadores de un equipo respecto a los de su rival, en una situació

Numérica

Igualdad

Numérica

Inferioridad

Numérica jugadores de un equipo respecto a los de

de juego ejecutada en una zona del terreno.

ejecutada en una zona del terreno.
Está determinada por la menor participación de jugadores de un equipo respecto a los de su rival, en un jug de un

Tabla 7

Dimensión. Zonas del Terreno de Juegó

\begin{tabular}{ll}
\hline Dimensión. Zonas del Terreno de Juego \\
\hline Defensiva Retrasada
\end{tabular}

Gestación Retrasada

Gestocion Aderasta

Gintion Ade

Finalización Adelantada

La jerarquización de las dimensiones se basó en la concreción del gol, razón por la que el rendimiento se constituye en el eje principal del análisis observacional, excluyendo así, las jugadas que no culminen en alguno de los criterios de esta dimensión (disparo, disparo a portería o gol), coincidiendo con el reglamento que confirma que obtendrá la victoria el equipo que consiga macar más veces en el arco adversario (Tenga, Ronglan \& Bahr, 2010).

\section{Procedimiento}

El proceso de diseño y validación de la construcción de la herramienta de observación para la valoración de acciones tácticas ofensivas en fútbol se llevó a cabo en 5 fases:

Primera Fase Revisión de literatura: Se realizó con el fin de definir las dimensiones del juego para comprender el contexto. Las bases inspeccionadas fueron Web of Science,
Google Scholar y Scopus. Las palabras claves en inglés y español fueron: «football», analytics, «soccer»y «observational instruments». Esto permitió desarrollar un instrumento borrador con las dimensiones y criterios a incluir con su respectiva definición (Ortega et al., 2019).

Segunda Fase Prueba piloto: se analizaron seis partidos de la selección de la Universidad de Pamplona (Colombia). Tres de la fase zonal y tres de la fase regional de los juegos de la Asociación Colombiana de Universidades (ASCUN) 2014 (Caicedo, Vera y Ortega, 2017). En esta fase se probó la primera versión de VATOF, para ajustar, debatir y fundamentar dimensiones del futbol de competición. La retroalimentación recibida sirvió como ajuste para la versión siguiente de VATOF.

Tercera Fase Validación del contenido y coherencia de VATOF: se utilizó la metodología DELPHI de juicio de expertos (Aranda et al., 2019). Los criterios de escogencia de los 10 jueces fueron: (a) ser doctor en Ciencias de la Actividad Física y Deporte, (b) 5 años de experiencia, (c) tener licencia de entrenador y haberlo sido durante mínimo 3 años.

Los jueces dieron su respuesta acerca de VATOF respecto de: a) Grado de pertinencia del objeto de estudio, b) Grado de comprensión de las dimensiones y criterios con su respectiva definición, c) Grado de congruencia de las dimensiones y criterios con su respectiva definición. La escala de valoración fue de 1 a 10, siendo 1 el límite inferior donde el juez no estuvo de acuerdo y 10 el límite superior o de máxima concordancia, conveniencia e importancia de cada uno de los ítems. Adicionalmente, se obtuvo una valoración cualitativa acerca de la d) Consideración de agregar o eliminar algún ítem. La validez del contenido de VATOF se determinó con el coeficiente de la V de Aiken (Aiken, 1985), ver (Tabla 8); evaluando el acuerdo entre los jueces sobre la validez del contenido (Soto \& Segovia, 2009).

Cuarta Fase Elaboración de VATOF: se construyó el diseño de VATOF, como instrumento de observación para la recolección de datos, (Castañer, Camerino \& Anguera, 2013) el cual quedó compuesto por 7 dimensiones y 40 criterios de análisis, con introducción a cambios y ajustes que fuesen necesarios. Este instrumento permite alcanzar una mayor precisión y consistencia del registro audio-visual de los datos y comportamientos observados.

Quinta Fase Entrenamiento en uso de VATOF y prueba de fiabilidad de la observación de los jueces: el observador se familiarizó con la herramienta en un primer momento, donde se le fueron explicadas las dimensiones y criterios del instrumento, en un segundo momento fue capacitado e instruido para detectar y codificar la acción e interacción de los jugadores. La confiabilidad del VATOF se realizó por medio del índice de kappa de Cohen, evaluación del nivel de acuerdo entre observadores (inter-observadores) y la fiabilidad (intra-observador) fue calculada a través del índice de kappa de Cohen, con el fin de probar validez y precisión del instrumento (Morillo et al., 2017).

\section{Aplicación}

Para facilitar la aplicación de VATOF, se diseñó un aplicativo informático (Figura 1). Esta app permite registrar el inicio y finalización de cada jugada, el cómo, cuándo y dónde se realizaron y el jugador que la ejecuta, cada registro 
inicia a partir del contacto con el balón y finaliza con la pérdida del mismo, donde cada secuencia o transición ofensiva se registra de acuerdo con los criterios expuestos. Cada usuario podrá controlar el registro de los acontecimientos por medio de comandos de voz, atajos del teclado o el uso del ratón, con datos precisos y relevantes que permiten obtener la frecuencia de las acciones registradas, actores principales en cada una de ellas y zonas del terreno de juego en que se ejecutan. Los datos obtenidos podrán ser exportados en diferentes aplicaciones y formatos de archivo para su análisis.

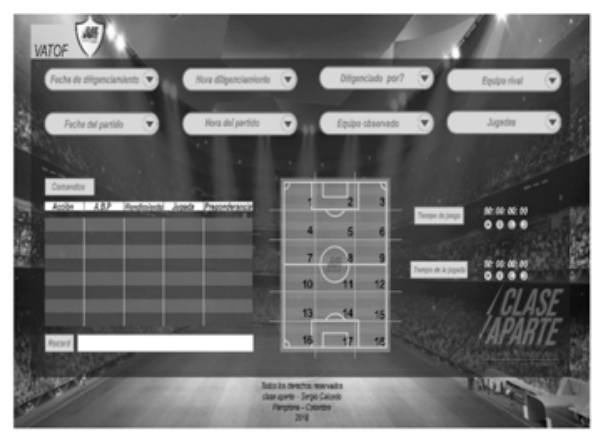

Figura 1. Interfaz de usuario de VATOF

De acuerdo con (Bakeman, McArthur, Quera \& Robinson, 1997) el componente más importante para el éxito de un estudio de observación sistemática es la elaboración de un instrumento de observación, el cual para su diseño debe surtir la siguientes etapas, elaboración del instrumento, registro o recolección de datos: análisis de datos e interpretación de resultados, garantizando la recolección de datos con la espontaneidad del comportamiento de los jugadores en el terreno y la naturalidad de cada una de las situaciones dadas en el desarrollo del juego (Tronchoni, Izquierdo y Anguera, 2018). Por esta razón los registros en el VATOF fueron realizados por un mismo evaluador, inician a partir del contacto con el balón y finaliza con la pérdida del mismo, donde cada secuencia o transición ofensiva se registra de acuerdo con los criterios expuestos.

\section{Resultados}

En el diseño del instrumento, las dimensiones y núcleos categóricos se seleccionaron siguiendo los núcleos categóricos de (Anguera, et al., 2011; Anguera \& Hernández-Mendo, 2015; García-López et al., 2013 \& Hernández-Mendo et al., 2014).

Tras la revisión del instrumento, las observaciones cualitativas de los expertos correspondieron con las definiciones de cada núcleo categórico, se muestra la lista núcleos categóricos obtenidos en el proceso de validación del instrumento. La validez de contenido se midió por el coeficiente V de Aiken. Los expertos no eliminaron ningún núcleo categórico después de la evaluación de los expertos. En la evaluación cuantitativa, todos los núcleos categóricos obtuvieron una puntuación media $>0.70$ (Tabla 8 ). El valor de $\mathrm{V}$ de Aiken más bajo encontrado fue de 0.80 , concretamente en el núcleo categórico «disparo».

Como resultado, los valores de kappa de Cohen oscilan en 1.00, lo que se interpreta como excelente por la fuerza del acuerdo, mostrando una alta confiablidad entre los observa- dores. Por otro lado, este análisis muestra como las dimensiones y núcleos categóricos presentan una confiabilidad alta, control y calidad del dato.

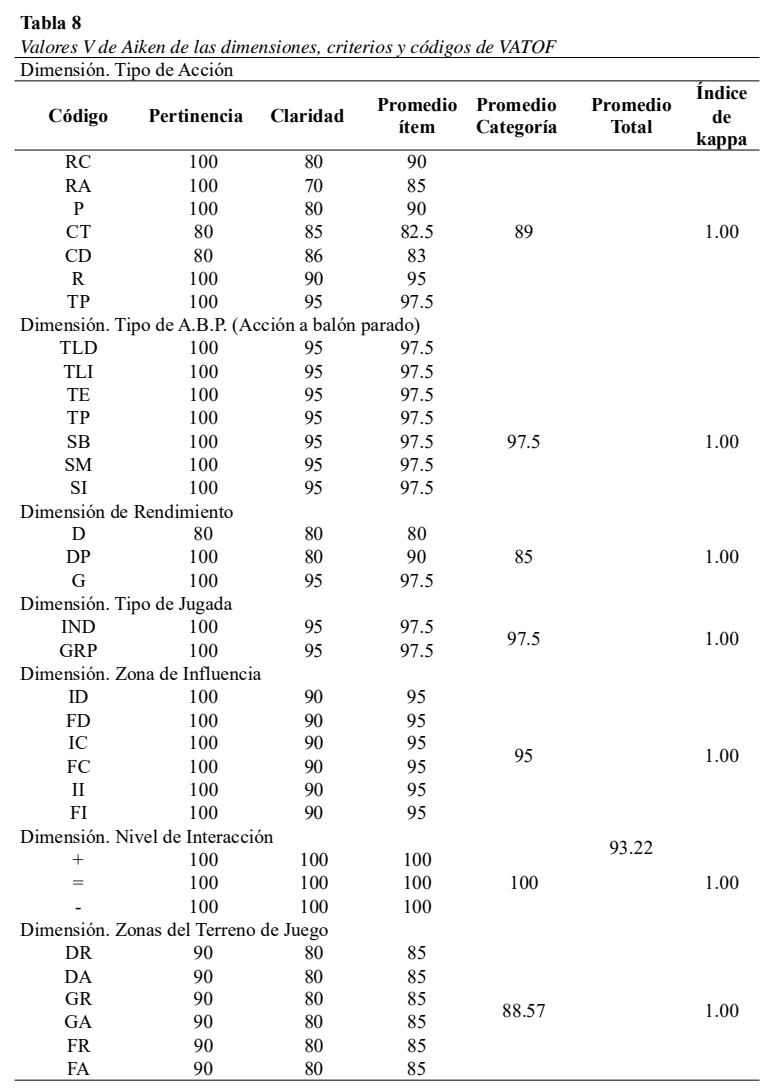

\section{Discusión}

En presente documento describe el proceso de construcción y validación de VATOF, herramienta práctica para la recolección de información clara y sistemática, permite definir parámetros de acuerdo a la obtención y control de los datos para su respectivo análisis (Anguera, Blanco-Villaseñor, Losada \& Portell, 2018), a la vez que cuantifica las acciones ejecutadas en periodos de tiempo determinados, permitiendo la evolución propia y adaptación al rival.

Este instrumento fue diseñado como un sistema de observación adaptado que permite analizar detalladamente cómo fueron ejecutadas las acciones tácticas ofensivas, mostrando que es una herramienta apropiada, equilibrada y pertinente para el análisis de las transiciones ofensivas, capaz de obtener información objetiva de los comportamientos tácticos con un sentido práctico en la aplicación de nuevos métodos de entrenamiento. La contextualización de cada uno de los parámetros proporciona suficiente información para evaluar los multieventos ofensivos. Además, el instrumento posee la secuencia progresiva del juego, registrando la acción desde la reanudación del juego o recuperación del móvil hasta la pérdida del mismo.

Asimismo, utiliza variables operacionales y conceptos que permiten describir la versatilidad y funcionalidad táctica del equipo observado como del adversario y de cada una de las acciones técnico-tácticas ejecutadas (McLean, Salmon, Gorman, Read \& Solomon, 2017); dato relevante en la interpretación de los comportamientos por los observadores, 
obteniendo niveles de discordancia más bajos. Como parámetro determinante, la dimensión de rendimiento es la que resume el éxito de la acción táctica ofensiva, manteniendo su interrelación con las otras dimensiones, pues en conjunto configuran el cómo, cuándo y dónde se ejecutan las jugadas.

Para la validez del contenido, se aplicó el cálculo del coeficiente $\mathrm{V}$ de Aiken, resumiendo las calificaciones de acuerdo con la opinión de cada experto, obteniendo un porcentaje del 93,22 de pertinencia y claridad del contenido con relación al objeto de estudio. La evaluación cuantitativa, obtuvo altos valores para todos los núcleos categóricos $(\mathrm{Vo}>0,70)$ y superiores a los valores mínimos propuestos en la literatura como referencia $(\mathrm{Vo}=0,70)$ por (Penfield \& Giacobbi; 2004). La evaluación cualitativa, utilizó definiciones operacionales que permiten describir la versatilidad y funcionalidad táctica de los diferentes núcleos categóricos; recurso sustancial para codificar de manera objetiva las particularidades de los comportamientos técnico-tácticos. Los expertos y el entrenamiento de los observadores, proporcionaron un panorama más amplio para comprender las características inherentes de los sistemas dinámicos y estructura de relación.

El análisis intraobservador, evidencia altos niveles de fiabilidad después de la capacitación y entrenamiento de los observadores (Bakeman et al., 1997). Lo que minimiza los errores de observación que pueden provenir del propio observador (Gamonales, Jiménez, León \& Ibáñez, 2018). El hecho puede corresponder a que los comportamientos requieren una interpretación de los observadores, lo que puede causar discordancias o disparidad entre los mismos (James et al., 2007; Losada \& Manolov, 2015), estos niveles de fiabilidad se obtuvieron con un periodo de observación de 3 semanas y definiciones operativas específicas al tema objeto de estudio.

Por tanto, se puede realizar una observación válida y fiable de las acciones tácticas a la ofensiva en jugadores de fútbol, identificando patrones de juego individuales y colectivos. Este instrumento tiene gran importancia en el entrenamiento deportivo ya que permite desarrollar estrategias y crear nuevas opciones sobre el rendimiento táctico ofensivo, generando una estructura organizativa con situaciones de superioridad que les permite anticiparse y crear sorpresa en el adversario.

\section{Conclusiones}

La herramienta de observación VATOF, manifiesta la validez del contenido, con niveles suficientemente altos para replicar en otros estudios, reflejando la participación activa de cada dimensión, proporcionando información significativa como los jugadores clave de acuerdo a su posición, ubicación y función dentro del terreno de juego y las zonas de mayor posesión y operatividad para marcar. Esta información permite a los entrenadores e investigadores un mayor conocimiento y dominio de los recursos que permitan desarrollar de manera exitosa la planificación del entrenamiento de acuerdo al objetivo propuesto.

\section{Agradecimientos}

Los autores agradecen el apoyo al canal RCN y Win
Sports por facilitar el material audiovisual y contribuir en el fortalecimiento científico y tecnológico sobre el análisis del rendimiento deportivo.

\section{Referencias}

Aiken, L. R. (1985). Three coefficients for analyzing the reliability and validity of ratings. Educational and psychological measurement, 45(1), 131-142. doi.org/10.1177/0013164485451012.

Anguera, M. T. (2003). Observational methods (general). Encyclopedia of psychological assessment, 2, 632-637.

Anguera, M. T., Blanco-Villaseñor, A., Losada, J. L. y Portell, M. (2018). Pautas para elaborar trabajos que utilizan la metodología observacional.Anuario de Psicología, 48(1), 9-17. doi.org/10.1016/ j.anpsic.2018.02.001

Anguera, A., Blanco, V., Hernández, M. y Losada, L. (2011). Observational designs: their suitability and application in sports psychology. Cuadernos de Psicología del Deporte, 11(2), 63-76.

Anguera, M. T., Blanco-Villaseñor,A., Losada, J. L.,Ardá, T., Camerino, O., Castellano, J. y Hernández-Mendo, A. (2003). Instrumento de codificación y registro de la acción de juego en fútbol (SOF-1). Revista Digital de Alto Rendimiento en Fútbol.

Anguera, M. T., Camerino, O., Castañer, M., Sánchez-Algarra, P. y Onwuegbuzie,A. J. (2017). The specificity of observational studies in physical activity and sports sciences: moving forward in mixed methods research and proposals for achieving quantitative and qualitative symmetry. Frontiers in psychology, 8, 2196. doi.org/ 10.3389/fpsyg.2017.02196.

Anguera, M. T. y Mendo, A. H. (2013). La metodología observacional en el ámbito del deporte. E-balonmano.com: Revista de Ciencias del Deporte, 2013, vol. 9, num. 3, p. 135-160.

Anguera, M. T. y Hernández, M. (2015). Data analysis techniques in observational studies in sport sciences. Cuadernos de Psicología del Deporte, 15(1), 13-29. doi.org/10.4321/S157884232015000100002.

Anguera, M. T., Portell, M., Chacón-Moscoso, S. y Sanduvete-Chaves, S. (2018). Indirect observation in everyday contexts: concepts and methodological guidelines within a mixed methods framework. Frontiers in psychology, 9, 13. doi.org/10.3389/fpsyg.2018.00013.

Aranda, R., González-Ródenas, J., López-Bondia, I., Aranda-Malavés, R., Tudela, A. y Anguera, M. T. (2019). «REOFUT» as an Observation Tool for Tactical Analysis on Offensive Performance in Soccer: Mixed Method Perspective. Frontiers in Psychology, 10, 1476. doi.org/10.3389/fpsyg.2019.01476.

Araújo, D., Teques, P., Hernández-Mendo, A., Reigal, R. y Anguera, M. T. (2016). La toma de decisión, ¿es una conducta observable?: Discusión sobre diferentes perspectivas teóricas utilizadas en el estudio del rendimiento deportivo. Cuadernos de psicología del deporte, 16(1), 183-196.

Bakeman, R., McArthur, D., Quera, V. y Robinson, B. F. (1997). Detecting sequential patterns and determining their reliability with fallible observers. Psychological Methods, 2(4), 357. doi.org/ 10.1037/1082-989X.2.4.357.

Ballesteros, J. L., Peñas, C. L., Eiras, E. R., Martinez, L. C. y Lago, E. D. (2012). El éxito ofensivo en el fútbol de élite: influencia de los modelos tácticos empleados y de las variables situacionales. European Journal of Human Movement, (28), 145-170.

Barreira, D., Garganta, J., Prudente, J., and Anguera, M. T. (2012). Desenvolvimento e validação de um sistema de observação aplicado à fase ofensiva em Futebol: Soccer Eye. Rev. Port. Ciênc. Desporto 12, 32-57. doi: 10.5628/rpcd.12.03.32.

Caicedo, S.A., Vera, J. L. y Ortega, A. J. (2017). Estudio de las acciones combinadas a la ofensiva en los futbolistas de la Universidad de Pamplona. Actividad física y desarrollo humano, 7(2). doi.org/ 10.24054/16927427.v2.n2.2016.2412.

Caicedo, S. A., Vera, J. L. y Ortega, A. J. (2018). Una nueva perspectiva 
de las acciones combinadas a la ofensiva y la toma de decisiones en el fútbol. Pamplona, Colombia, Editorial Universidad de Pamplona.

Castañer Balcells, M., Camerino Foguet, O. y Anguera Argilaga, M. T. (2013). Métodos mixtos en la investigación de las ciencias de la actividad física y el deporte. Apunts. Educació Física I Esports, 2013, vol. 112, num. 2, p. 31-36. doi.org/10.5672/apunts.20140983.es.(2013/2).112.01

Castellano, J. (2000). Observación y Análisis de la Acción de Juego en Fútbol. Ph.D. thesis, University of the Basque Country, Álava.

Chacón-Moscoso, S., Sanduvete-Chaves, S., Anguera, M. T., Losada, J. L., Portell, M. y Lozano-Lozano, J. A. (2018). Preliminary checklist for reporting observational studies in sports areas: content validity. Frontiers in psychology, 9, 291. doi.org/10.3389/ fpsyg.2018.00291.

Costa, I. T., Garganta, J., Greco, P. J., Mesquita, I. y Maia, J. (2011) System of tactical assessment in Soccer (FUT-SAT): Development and preliminary validation. System, 7(1), 69-83. doi:10.6063/ motricidade.7(1).121.

Cuerva, E. M. y Ruano, M. A. G. (2017). Validación herramienta observacional para el análisis de rachas de lanzamiento en baloncesto. Revista de psicología del deporte, 26(1), 87-93.

Davids, K. W., Button, C. y Bennett, S. J. (2008). Dynamics of skill acquisition: A constraints-led approach. Human Kinetics.

Dios, R. M., Jiménez, M. A., García, J. E. M. y García, S. L. (2018). Análisis observacional de las relaciones interlíneas de la Selección Española de Fútbol, mediante coordenadas polares. Cuadernos de Psicología del Deporte, 18(2), 18-32.

Dufour, M., Phillips, J. y Ernwein, V. (2017). What makes the difference? Analysis of the 2014 World Cup. Journal of Human Sport and Exercise, 12(3), 616-629. doi:10.14198/jhse.2017.123.06.

Fabra, P., Balaguer, I., Tomás, I., Smith, N. y Duda, J. L. (2018). Versión española del Sistema de Observación del Clima Motivacional Multidimensional (MMCOS): fiabilidad y evidencias de validez. Rev. Psicol. Deporte, 27, 11-22.

Gamonales, J., Jiménez, J., León, K., e Ibáñez, S. (2018). Entrenamiento y confiabilidad entre observadores en el análisis del fútbol para ciegos. Retos: nuevas tendencias en educación física, deporte y recreación, (34), 155-161.

García López, L. M., González Víllora, S., Gutiérrez, D. y Serra, J. (2013). Development and validation of the Game Performance Evaluation Tool (GPET) in soccer. Sport TK, vol. 2, nº 1 (2013).

González Rodenas, J. (2013). Análisis del rendimiento táctico en el juego colectivo ofensivo en fútbol en la copa del mundo 2010. (Tesis doctoral). Universitat de València. Valencia.

Gréhaigne, J. F. y Godbout, P. (2014). Dynamic systems theory and team sport coaching. Quest, 66(1), 96-116.

Gudmundsson, J. y Horton, M. (2017). Spatio-temporal analysis of team sports. ACM Computing Surveys (CSUR), 50(2), 22. doi: $10.1145 / 3054132$

Hernández-Mendo, A. H., Paulis, J. C., Camerino, O., Jonsson, G., Blanco-Villasenor, A. y Argilaga, M. T. A. (2014). Observational software, data quality control and data analysis. Revista de Psicología del Deporte, 23(1), 111-121.

Hughes, M. (2003). «Notational analysis,» in Science and Soccer, eds T. Reilly and A. M.Williams (London: Routledge), 245-263.

James, N., Taylor, J., and Stanley, S. (2007). Reliability procedures for categorical data in performance analysis. International Journal of Performance Analysis in Sport, 7(1), 1-11. doi.org/10.1080/ 24748668.2007.11868382.

Lago-Ballesteros, J. y Lago-Peñas, C. (2010). Performance in team sports: Identifying the keys to success in soccer. Journal of Human Kinetics, 25, 85-91. doi10.2478/v10078-010-0035-0.

Losada, J. L. y Manolov, R. (2015). The process of basic training, applied training, maintaining the performance of an observer. Quality y Quantity, 49(1), 339-347. doi.org/10.1007/s11135-0149989-7.

Lozano, D., Camerino, O. y Hileno, R. (2016). Análisis del comportamiento táctico ofensivo en momentos críticos de juego en el alto rendimiento en balonmano: un estudio Mixed Methods. Cuadernos de Psicología del Deporte, 16(1), 151-160.

Mackenzie, R. y Cushion, C. (2013). Performance analysis in football: A critical review and implications for future research. Journal of sports sciences, 31(6), 639-676. doi.org/10.1080/ 02640414.2012.746720.

Maneiro, R., Losada López, J. L., Casal, C. A., \& Ardá Suárez, A. (2017). Multivariate analysis of indirect free kick in the FIFA World Cup 2014. Anales de Psicología, 2017, vol. 33, num. 3, p. 461-470. doi.org/10.6018/analesps.33.3.271031.

McLean, S., Salmon, P. M., Gorman, A. D., Read, G. J. y Solomon, C. (2017). What's in a game? A systems approach to enhancing performance analysis in football. PloS one, 12(2), e0172565. doi.org/ 10.1371/journal.pone.0172565.

Morillo, J. P., Reigal, R. E., Hernández-Mendo, A., Montaña, A. y Morales-Sánchez, V.(2017). Decision-making by handball referees: design of an ad hoc observation instrument and polar coordinate analysis. Frontiers in psychology, 8, 1842. doi.org/10.3389/ fpsyg.2017.01842.

Nadal Comas, G., Serna Bardavío, J., Nuviala Nuviala, R. y Falcón Miguel, D. (2018). Diseño de un instrumento observacional para la valoración del penalti en fútbol y análisis de los resultados obtenidos. Revista de Psicología del Deporte, 2018, vol. 27, núm 2, p. 189-199.

Ortega-Toro, E., García-Angulo, A., Giménez-Egido, J. M., GarcíaAngulo, F. J. y Palao, J. M.(2019). Design, Validation, and Reliability of an Observation Instrument for Technical and Tactical Actions of the Offense Phase in Soccer. Frontiers in psychology, 10, 22. doi.org/ 10.3389/fpsyg.2019.00022.

Penfield, R. D. y Giacobbi, Jr, P. R. (2004). Applying a score confidence interval to Aiken's item content-relevance index. Measurement in Physical Education and Exercise Science, 8(4), 213-225. doi.org/ 10.1207/s15327841mpee0804 3.

Sabio Lago, Y., Guerra Balic, M., Cabedo Sanromà, J., Solà Santesmases, J., \& Argudo Iturriaga, F. (2018). Diseño, validación y fiabilidad de un instrumento para analizar acciones técnico-tácticas en waterpolo Design, validation and reliability of an instrument to analyze technical-tactical actions in water polo. Retos.

Sánchez-Algarra, P. y Anguera, M. T. (2013). Qualitative/quantitative integration in the inductive observational study of interactive behaviour: impact of recording and coding among predominating perspectives. Quality y Quantity, 47(2), 1237-1257. DOI 10.1007/ s11135-012-9764-6.

Sarmento, H., Bradley, P., Anguera, M. T., Polido, T., Resende, R. y Campaniço, J. (2016). Quantifying the offensive sequences that result in goals in elite futsal matches. Journal of sports sciences, 34(7), 621-629. doi.org/10.1080/02640414.2015.1066024.

Sarmento, H., Clemente, F. M., Araújo, D., Davids, K., McRobert, A. y Figueiredo, A. (2018). What performance analysts need to know about research trends in association football (2012-2016): A systematic review. Sports medicine, 48(4), 799-836. doi.org/ 10.1007/s40279-017-0836-6

Serna Bardavío, J., MuñozArroyave, V., Hileno González, R., Solsona Leri, E. y Sáez de Ocáriz Granja, U. (2017). Patrones temporales iniciados con bloqueo directo o uno contra uno en baloncesto. Revista de psicología del deporte, 26(3), 0081-86.

Soto, C. M. y Segovia, J. L. (2009). Intervalos de confianza asimétricos para el índice la validez de contenido: Un programa Visual Basic para la V de Aiken. Anales de Psicología/Annals of Psychology, 25(1), 169-171.

Tenga,A., Ronglan, L. T. y Bahr, R. (2010). Measuring the effectiveness of offensive match-play in professional soccer. European Journal of Sport Science, 10(4), 269-277. doi.org/10.1080/ 17461390903515170.

Travassos, B., Davids, K., Araújo, D. y Esteves, T. P. (2013). Performance analysis in team sports: Advances from an Ecological Dynamics approach. International Journal of Performance Analysis in Sport, 13(1), 83-95. doi.org/10.1080/247486 